\title{
A Hilbert-Type Inequality with Some Parameters and the Integral in Whole Plane
}

\author{
Zitian Xie ${ }^{1}$, Zheng Zeng ${ }^{2}$ \\ ${ }^{1}$ Department of Mathematics, Zhaoqing University, Zhaoqing, China \\ ${ }^{2}$ Department of Mathematics, Shaoguan University, Shaoguan, China \\ E-mail: gdzqxzt@163.com,zz@sgu.edu.cn
}

Received February 13, 2011; revised March 7, 2011; accepted March 10, 2011

\begin{abstract}
In this paper, by introducing some parameters and estimating the weight coefficient, we give a new Hilbert's inequality with the integral in whole plane and with a non-homogeneous and the equivalent form is given as well. The best constant factor is calculated by the way of Complex Analysis.
\end{abstract}

Keywords: Hilbert-Type Integral Inequality, Weight Function, Holder's Inequality

\section{Introduction}

If $f(x), g(x)$ are non-negative functions such that $0<\int_{0}^{\infty} f^{2}(x) \mathrm{d} x<\infty$ and $0<\int_{0}^{\infty} g^{2}(x) \mathrm{d} x<\infty$, then

$$
\int_{0}^{\infty} \int_{0}^{\infty} \frac{f(x) g(y)}{x+y} \mathrm{~d} x \mathrm{~d} y<\pi\left\{\int_{0}^{\infty} f(x) \mathrm{d} x \int_{0}^{\infty} g(x) \mathrm{d} x\right\}^{1 / 2}
$$

where the constant factor $\pi$ is the best possible. Inequality (1.1) is well-known as Hilbert's integral inequality, which has been extended by Hardy-Riesz as [2]:

If $p>1, \frac{1}{p}+\frac{1}{q}=1, f(x), g(x) \geq 0$, such that $0<\int_{0}^{\infty} f^{p}(x) \mathrm{d} x<\infty$ and $0<\int_{0}^{\infty} g^{q}(x) \mathrm{d} x<\infty$, then we have the following Hardy-Hilbert's integral inequality:

$$
\begin{aligned}
& \int_{0}^{\infty} \int_{0}^{\infty} \frac{f(x) g(y)}{x+y} \mathrm{~d} x \mathrm{~d} y \\
& <\frac{\pi}{\sin (\pi / p)}\left(\int_{0}^{\infty} f^{p}(x) \mathrm{d} x\right)^{1 / p}\left(\int_{0}^{\infty} g^{q}(x) \mathrm{d} x\right)^{1 / q}
\end{aligned}
$$

where the constant factor $\frac{\pi}{\sin (\pi / p)}$ also is the best possible.

In recent years, by introducing some parameters and estimating the way of weight function, inequalities (1.1) and (1.2) have many generalizations and variants (1.1) has been strengthened by Yang and others. (including double series inequalities) [3-15].

In 2005 Yang gave a Hilbert-type Inequality [3] as follows: If $p>1, \quad \frac{1}{p}+\frac{1}{q}=1, f(x), g(x) \geq 0$, $2-\min \{p, q\}<\lambda<1, \quad 0<\int_{0}^{\infty} x^{1-\lambda} f^{p}(x) \mathrm{d} x<\infty, \quad$ and $0<\int_{0}^{\infty} x^{1-\lambda} g^{q}(x) \mathrm{d} x<\infty$ then

$$
\begin{aligned}
& \int_{0}^{\infty} \int_{0}^{\infty} \frac{f(x) g(y)}{|x-y|^{\lambda}} \mathrm{d} x \mathrm{~d} y \\
& <K\left(\int_{0}^{\infty} x^{1-\lambda} f^{p}(x) \mathrm{d} x\right)^{1 / p}\left(\int_{0}^{\infty} x^{1-\lambda} g^{q}(x) \mathrm{d} x\right)^{1 / q}
\end{aligned}
$$

where the constant factor

$K=\left[B\left(\frac{p+\lambda-2}{p}, 1-\lambda\right)+B\left(\frac{q+\lambda-2}{q}, 1-\lambda\right)\right]$ also is the best possible.

In 2008 Xie gave a new Hilbert-type Inequality [4] as follows:

$$
\begin{aligned}
& \text { If } p>1, \frac{1}{p}+\frac{1}{q}=1, f(x), g(x) \geq 0 \\
& 0<\int_{0}^{\infty} x^{1-p / 2} f^{p}(x) \mathrm{d} x<\infty \text { and } 0<\int_{0}^{\infty} x^{1-q / 2} g^{q}(x) \mathrm{d} x<\infty,
\end{aligned}
$$
then

$$
\begin{aligned}
& \int_{0}^{\infty} \int_{0}^{\infty} \frac{f(x) g(y)}{\left(x+a^{2} y\right)\left(x+a^{2} y\right)\left(x+a^{2} y\right)} \mathrm{d} x \mathrm{~d} y \\
& <K\left(\int_{0}^{\infty} x^{1-p / 2} f^{p}(x) \mathrm{d} x\right)^{1 / p}\left(\int_{0}^{\infty} x^{1-q / 2} g^{q}(x) \mathrm{d} x\right)^{1 / q}
\end{aligned}
$$

where the constant factor $K=\frac{\pi}{(a+b)(b+c)(c+a)}$ 
also is the best possible.

In this paper, by using the way of weight function and the technic of real analysis and by the way of complex analysis, a new Hilbert-type inequality with the integral in whole plane is given.

In the following, we always suppose that: $p>0$, $\frac{1}{p}+\frac{1}{q}=1, \quad p \neq 1, \quad r \in(1,2), 0 \leq \alpha<\beta \leq \pi$.

\section{Some Lemmas}

\section{Lemma 2.1 If}

$$
\begin{aligned}
& k_{1}:=\int_{0}^{\infty} u^{-r} \ln \frac{1+2 u \cos \alpha+u^{2}}{1+2 u \cos \beta+u^{2}} \mathrm{~d} u \\
& k_{2}:=\int_{0}^{\infty} u^{-r} \ln \frac{1-2 u \cos \beta+u^{2}}{1-2 u \cos \alpha+u^{2}} \mathrm{~d} u
\end{aligned}
$$

then

$$
\begin{aligned}
& k_{1}:=\frac{4 \pi \sin \frac{(1-r)(\beta-\alpha)}{2} \sin \frac{(1-r)(\beta+\alpha)}{2}}{(1-r) \sin r \pi}, \\
& k_{2}:=\frac{4 \pi \sin \frac{(1-r)(\beta-\alpha)}{2} \sin \left[(1-r)\left(\pi-\frac{\beta+\alpha}{2}\right)\right]}{(1-r) \sin r \pi}
\end{aligned}
$$

and

$$
\begin{aligned}
k & :=\int_{-\infty}^{\infty}|u|^{-r}\left|\ln \frac{1+2 u \cos \alpha+u^{2}}{1+2 u \cos \beta+u^{2}}\right| \mathrm{d} u \\
& =k_{1}+k_{2} \\
& =\frac{4 \pi \sin \frac{(1-r)(\beta-\alpha)}{2} \cos \frac{(1-r)(\pi-\beta-\alpha)}{2}}{(1-r) \sin \frac{r \pi}{2}}
\end{aligned}
$$

Proof We have

$$
\begin{aligned}
A:= & \int_{0}^{\infty} x^{-r} \ln \left(x^{2}+2 x \cos \alpha+1\right) \mathrm{d} x \\
= & \left.\frac{1}{1-r} x^{1-r} \ln \left(x^{2}+2 x \cos \alpha+1\right)\right|_{0} ^{\infty} \\
& -\frac{2}{1-r} \int_{0}^{\infty} \frac{x^{1-r}(x+\cos \alpha)}{x^{2}+2 x \cos \alpha+1} \mathrm{~d} x \\
:= & -\frac{2}{1-r} B
\end{aligned}
$$

setting $f(z)=\frac{z^{1-r}(z+\cos \alpha)}{z^{2}+2 z \cos \alpha+1}, \quad z_{1}=-e^{i \alpha}, z_{2}=-e^{-i \alpha}$, then

$$
\begin{aligned}
B & =\frac{2 \pi i}{1-e^{2 \pi r i}}\left[\operatorname{Res}\left(f, z_{1}\right)+\operatorname{Res}\left(f, z_{2}\right)\right] \\
& =\frac{2 \pi i}{1-e^{2 \pi r i}}\left[\frac{z_{1}^{1-r}\left(z_{1}+\cos \alpha\right)}{z_{1}-z_{2}}+\frac{z_{2}^{1-r}\left(z_{2}+\cos \alpha\right)}{z_{2}-z_{1}}\right] \\
& =-\frac{\pi \cos (1-r) \alpha}{\sin r \pi}
\end{aligned}
$$

We find $A=-\frac{2}{1-r} \quad B=\frac{2 \pi \cos [(1-r) \alpha]}{(1-r) \sin r \pi}$.

then

$$
\begin{aligned}
k_{1} & =\int_{0}^{\infty} u^{-r} \ln \frac{1+2 u \cos \alpha+u^{2}}{1+2 u \cos \beta+u^{2}} \mathrm{~d} u \\
& =\frac{4 \pi \sin \frac{(1-r)(\beta-\alpha)}{2} \sin \frac{(1-r)(\beta+\alpha)}{2}}{(1-r) \sin r \pi}
\end{aligned}
$$

and

$$
\begin{aligned}
k_{2} & =\int_{0}^{\infty} u^{-r} \ln \frac{1-2 u \cos \beta+u^{2}}{1-2 u \cos \alpha+u^{2}} \mathrm{~d} u \\
& =\int_{0}^{\infty} u^{-r} \ln \frac{1+2 u \cos (\pi-\beta)+u^{2}}{1+2 u \cos (\pi-\alpha)+u^{2}} \mathrm{~d} u \\
& =\frac{4 \pi \sin \frac{(1-r)(\beta-\alpha)}{2} \sin \left[(1-r)\left(\pi-\frac{\beta+\alpha}{2}\right)\right]}{(1-r) \sin r \pi}
\end{aligned}
$$

$$
\begin{aligned}
k= & \int_{-\infty}^{\infty}|u|^{-r}\left|\ln \frac{1+2 u \cos \alpha+u^{2}}{1+2 u \cos \beta+u^{2}}\right| \mathrm{d} u \\
= & \int_{0}^{\infty} u^{-r} \ln \frac{1+2 u \cos \alpha+u^{2}}{1+2 u \cos \beta+u^{2}} \mathrm{~d} u \\
& +\int_{-\infty}^{0}(-u)^{-r} \ln \frac{1+2 u \cos \beta+u^{2}}{1+2 u \cos \alpha+u^{2}} \mathrm{~d} u \\
= & k_{1}+k_{2} \\
= & \frac{4 \pi \sin \frac{(1-r)(\beta-\alpha)}{2} \cos \frac{(1-r)(\pi-\beta-\alpha)}{2}}{(1-r) \sin \frac{r \pi}{2}}
\end{aligned}
$$

The lemma is proved.

Lemma 2.2 Define the weight functions as follow:

$$
\begin{aligned}
& w(x):=\int_{-\infty}^{\infty} \frac{|x|^{-r+1}}{|y|^{r}}\left|\ln \frac{1+2 x y \cos \alpha+x^{2} y^{2}}{1+2 x y \cos \beta+x^{2} y^{2}}\right| \mathrm{d} y, \\
& \tilde{w}(x):=\int_{-\infty}^{\infty} \frac{|y|^{-r+1}}{|x|^{r}}\left|\ln \frac{1+2 x y \cos \alpha+x^{2} y^{2}}{1+2 x y \cos \beta+x^{2} y^{2}}\right| \mathrm{d} x
\end{aligned}
$$

then 


$$
\begin{aligned}
& w(x)=\tilde{w}(x)=k \\
& =\frac{4 \pi \sin \frac{(1-r)(\beta-\alpha)}{2} \cos \frac{(1-r)(\pi-\beta-\alpha)}{2}}{(1-r) \sin \frac{r \pi}{2}}
\end{aligned}
$$

Proof We only prove that $w(x)=k$ for $x \in(-\infty, 0)$.

$$
\begin{aligned}
w(x)= & \int_{-\infty}^{0} \frac{|x|^{-r+1}}{|y|^{r}}\left|\ln \frac{1+2 x y \cos \alpha+x^{2} y^{2}}{1+2 x y \cos \beta+x^{2} y^{2}}\right| \mathrm{d} y \\
& +\int_{0}^{\infty} \frac{|x|^{-r+1}}{|y|^{r}}\left|\ln \frac{1+2 x y \cos \alpha+x^{2} y^{2}}{1+2 x y \cos \beta+x^{2} y^{2}}\right| \mathrm{d} y \\
= & \int_{-\infty}^{0} \frac{(-x)^{-r+1}}{(-y)^{r}} \ln \frac{1+2 x y \cos \alpha+x^{2} y^{2}}{1+2 x y \cos \beta+x^{2} y^{2}} \mathrm{~d} y \\
& +\int_{0}^{\infty} \frac{(-x)^{-r+1}}{y^{r}} \ln \frac{1+2 x y \cos \beta+x^{2} y^{2}}{1+2 x y \cos \alpha+x^{2} y^{2}} \mathrm{~d} y \\
= & w_{1}+w_{2}
\end{aligned}
$$

Setting $u=x y$, then

$$
\begin{aligned}
& w_{1}=\int_{-\infty}^{0} \frac{(-x)^{-r+1}}{(-y)^{r}} \ln \frac{1+2 x y \cos \alpha+x^{2} y^{2}}{1+2 x y \cos \beta+x^{2} y^{2}} d y \\
& =\int_{0}^{\infty} u^{-r} \ln \frac{1+2 u \cos \alpha+u^{2}}{1+2 u \cos \beta+u^{2}} d u=k_{1}
\end{aligned}
$$

Similarly, setting $u=-x y$,

$$
w_{2}=\int_{0}^{\infty} u^{-r} \ln \frac{1-2 u \cos \beta+u^{2}}{1-2 u \cos \alpha+u^{2}} \mathrm{~d} u=k_{2}
$$

And $w(x)=k_{1}+k_{2}=k$, we have (2.3).

Lemma 2.3 For $0<\varepsilon$, and $\left(r+\max \left\{\frac{2 \varepsilon}{p}, \frac{2 \varepsilon}{q}\right\}\right) \in(1,2)$, define both functions, f and $g$ as follow:

$$
f(x)= \begin{cases}x^{-r-2 \varepsilon / p}, & \text { if } x \in(1, \infty), \\ 0, & \text { if } x \in[-1,1] \\ (-x)^{-r-2 \varepsilon / p}, & \text { if } x \in(-\infty, 1)\end{cases}
$$

and

$$
g(x)= \begin{cases}0, & \text { if } x \in(1, \infty), \\ |x|^{-r+2 \varepsilon / q}, & \text { if } x \in[-1,1], \\ 0, & \text { if } x \in(-\infty, 1) ;\end{cases}
$$

then

$$
\begin{aligned}
I(x) & :=2 \varepsilon\left\{\int_{-\infty}^{\infty}|x|^{-1} f^{p}(x) \mathrm{d} x\right\}^{1 / p}\left\{\int_{-\infty}^{\infty}|x|^{-1} g^{q}(x) \mathrm{d} x\right\}^{1 / p}=1 \\
\tilde{I}(\varepsilon) & :=2 \varepsilon \int_{-\infty}^{\infty} \int_{-\infty}^{\infty} f(x) g(y)\left|\ln \frac{1+2 x y \cos \alpha+x^{2} y^{2}}{1+2 x y \cos \beta+x^{2} y^{2}}\right| \mathrm{d} x \mathrm{~d} y \\
& =k+o(1), \quad\left(\text { for } \varepsilon \rightarrow 0^{+}\right)
\end{aligned}
$$

Proof Easily,

$$
I(x):=2 \varepsilon\left\{\int_{1}^{\infty} x^{-1} x^{-2 \varepsilon} \mathrm{d} x\right\}^{1 / p}\left\{\int_{0}^{1} x^{-1} x^{-2 \varepsilon} \mathrm{d} x\right\}^{1 / p}=1 ;
$$

Let $y=-Y$, using $\not f(-x)=f(x), g(-x)=g(x)$, and

$$
\begin{aligned}
& f(-x) \int_{-\infty}^{\infty} g(y)\left|\ln \frac{1-2 x y \cos \alpha+x^{2} y^{2}}{1-2 x y \cos \beta+x^{2} y^{2}}\right| \mathrm{d} y \\
& =f(x) \int_{-\infty}^{\infty} g(Y)\left|\ln \frac{1+2 x Y \cos \alpha+x^{2} Y^{2}}{1+2 x Y \cos \beta+x^{2} Y^{2}}\right| \mathrm{d} Y
\end{aligned}
$$

we have that $f(x) \int_{-\infty}^{\infty} g(y)\left|\ln \frac{1+2 x y \cos \alpha+x^{2} y^{2}}{1+2 x y \cos \beta+x^{2} y^{2}}\right| d y$ is an even function, then

$$
\begin{aligned}
& \tilde{I}(\varepsilon)=2 \varepsilon \int_{0}^{\infty} f(x)\left(\int_{-\infty}^{\infty} g(y)\left|\ln \frac{1+2 x y \cos \alpha+x^{2} y^{2}}{1+2 x y \cos \beta+x^{2} y^{2}}\right| \mathrm{d} y\right) \mathrm{d} x \\
& =2 \varepsilon\left[\int_{1}^{\infty} x^{-r-2 \varepsilon / p}\left(\int_{-1}^{0}(-y)^{-r+2 \varepsilon / q}\left|\ln \frac{1+2 x y \cos \alpha+x^{2} y^{2}}{1+2 x y \cos \beta+x^{2} y^{2}}\right| \mathrm{d} y\right) \mathrm{d} x+\int_{1}^{\infty} x^{-r-2 \varepsilon / p}\left(\int_{0}^{1} y^{-r+2 \varepsilon / q}\left|\ln \frac{1+2 x y \cos \alpha+x^{2} y^{2}}{1+2 x y \cos \beta+x^{2} y^{2}}\right| \mathrm{d} y\right) \mathrm{d} x\right] \\
& =I_{1}+I_{2} .
\end{aligned}
$$

Setting $u=x y$ then

$$
\begin{aligned}
I_{1} & =2 \varepsilon \int_{1}^{\infty} x^{-r-2 \varepsilon / p}\left(\int_{0}^{1} y^{-r+2 \varepsilon / q} \ln \frac{1-2 x y \cos \beta+x^{2} y^{2}}{1-2 x y \cos \alpha+x^{2} y^{2}} \mathrm{~d} y\right) \mathrm{d} x=2 \varepsilon \int_{1}^{\infty} x^{-1-2 \varepsilon}\left(\int_{0}^{x} u^{-r+2 \varepsilon / q} \ln \frac{1-2 u \cos \beta+u^{2}}{1-2 u \cos \alpha+u^{2}} \mathrm{~d} u\right) \mathrm{d} x \\
& =2 \varepsilon\left[\int_{1}^{\infty} x^{-1-2 \varepsilon}\left(\int_{0}^{1} u^{-r+2 \varepsilon / q} \ln \frac{1-2 u \cos \beta+u^{2}}{1-2 u \cos \alpha+u^{2}} \mathrm{~d} u\right) \mathrm{d} x+\int_{1}^{\infty} x^{-1-2 \varepsilon}\left(\int_{1}^{x} u^{-r+2 \varepsilon / q} \ln \frac{1-2 u \cos \beta+u^{2}}{1-2 u \cos \alpha+u^{2}} \mathrm{~d} u\right) \mathrm{d} x\right]
\end{aligned}
$$




$$
\begin{aligned}
& =\int_{0}^{1} u^{-r+2 \varepsilon / q} \ln \frac{1-2 u \cos \beta+u^{2}}{1-2 u \cos \alpha+u^{2}} \mathrm{~d} u+2 \varepsilon \int_{1}^{\infty} u^{-r+2 \varepsilon / q} \ln \frac{1-2 u \cos \beta+u^{2}}{1-2 u \cos \alpha+u^{2}}\left(\int_{u}^{\infty} x^{-1-2 \varepsilon} \mathrm{d} x\right) \mathrm{d} u \\
& =\int_{0}^{1} u^{-r+2 \varepsilon / q} \ln \frac{1-2 u \cos \beta+u^{2}}{1-2 u \cos \alpha+u^{2}} \mathrm{~d} u+\int_{1}^{\infty} u^{-r-2 \varepsilon / p} \ln \frac{1-2 u \cos \beta+u^{2}}{1-2 u \cos \alpha+u^{2}} \mathrm{~d} u \\
& =\int_{0}^{\infty} u^{-r-2 \varepsilon / p} \ln \frac{1-2 u \cos \beta+u^{2}}{1-2 u \cos \alpha+u^{2}} \mathrm{~d} u+\int_{0}^{1}\left(u^{2 \varepsilon / q}-u^{-2 \varepsilon / p}\right) u^{-r} \ln \frac{1-2 u \cos \beta+u^{2}}{1-2 u \cos \alpha+u^{2}} \mathrm{~d} u \\
& =\frac{4 \pi \sin \frac{\left(1-r-\frac{2 \varepsilon}{p}\right)(\beta-\alpha)}{2} \sin \left[\left(1-r-\frac{2 \varepsilon}{p}\right)\left(\pi-\frac{\beta+\alpha}{2}\right)\right]}{\left(1-r-\frac{2 \varepsilon}{p}\right) \sin \left[\frac{1}{2}\left(r+\frac{2 \varepsilon}{p}\right) \pi\right]}+\eta(\varepsilon)
\end{aligned}
$$

There $\lim _{\varepsilon \rightarrow 0^{+}} \eta(\varepsilon)=0$, and we have $I_{1} \rightarrow k_{2}$ (for $\left.\varepsilon \rightarrow 0^{+}\right)$

Similarly $I_{2} \rightarrow k_{1} \quad\left(\right.$ for $\varepsilon \rightarrow 0^{+}$). The lemma is proved.

Lemma 2.4 If $0<\int_{-\infty}^{\infty}|x|^{p r-1} f^{p}(x) \mathrm{d} x<\infty$, we have

$$
\begin{aligned}
J & :=\int_{-\infty}^{\infty}|y|^{-p r+p-1}\left(\int_{-\infty}^{\infty} f(x)\left|\ln \frac{1+2 x y \cos \alpha+x^{2} y^{2}}{1+2 x y \cos \beta+x^{2} y^{2}}\right| \mathrm{d} x\right)^{p} \mathrm{~d} y \\
& \leq k^{p} \int_{-\infty}^{\infty}|x|^{p r-1} f^{p}(x) \mathrm{d} x
\end{aligned}
$$

Proof By lemma 2.2, we find

$$
\begin{aligned}
& \left(\int_{-\infty}^{\infty} f(x)\left|\ln \frac{1+2 x y \cos \alpha+x^{2} y^{2}}{1+2 x y \cos \beta+x^{2} y^{2}}\right| \mathrm{d} x\right)^{p} \\
& =\left(\int_{-\infty}^{\infty}\left|\ln \frac{1+2 x y \cos \alpha+x^{2} y^{2}}{1+2 x y \cos \beta+x^{2} y^{2}}\right|\left(\frac{|x|^{r / q}}{|y|^{r / p}} f(x)\right)\left(\frac{|y|^{r / p}}{|x|^{r / q}}\right) \mathrm{d} x\right)^{p} \\
& \leq \int_{-\infty}^{\infty}\left|\ln \frac{1+2 x y \cos \alpha+x^{2} y^{2}}{1+2 x y \cos \beta+x^{2} y^{2}}\right| \frac{|x|^{r(p-1)}}{|y|^{r}} f^{p}(x) \mathrm{d} x \\
& \left(\int_{-\infty}^{\infty}\left|\ln \frac{1+2 x y \cos \alpha+x^{2} y^{2}}{1+2 x y \cos \beta+x^{2} y^{2}}\right| \frac{|y|^{r(q-1)}}{|x|^{r}} \mathrm{~d} x\right)^{p-1} \\
& =k^{p-1}|y|^{p r-p+1} \int_{-\infty}^{\infty}\left|\ln \frac{1+2 x y \cos \alpha+x^{2} y^{2}}{1+2 x y \cos \beta+x^{2} y^{2}}\right| \frac{|x|^{r(p-1)}}{|y|^{r}} f^{p}(x) \mathrm{d} x, \\
& J \\
& \leq k^{p-1} \int_{-\infty}^{\infty}\left[\int_{-\infty}^{\infty}\left|\ln \frac{1+2 x y \cos \alpha+x^{2} y^{2}}{1+2 x y \cos \beta+x^{2} y^{2}}\right| \frac{|x|^{r(p-1)}}{|y|^{r}} f^{p}(x) \mathrm{d} x\right] \mathrm{d} y \\
& =k^{p-1} \int_{-\infty}^{\infty}\left[\int_{-\infty}^{\infty}\left|\ln \frac{1+2 x y \cos \alpha+x^{2} y^{2}}{1+2 x y \cos \beta+x^{2} y^{2}}\right| \frac{|x|^{r(p-1)}}{|y|^{r}} \mathrm{~d} y\right] f^{p}(x) \mathrm{d} x \\
& =k^{p} \int_{-\infty}^{\infty}|x|^{p r-1} f^{p}(x) \mathrm{d} x
\end{aligned}
$$

\section{Main Results}

Theorem If both functions, $f(x)$ and $g(x)$ are nonnegative measurable functions, and satisfy $0<\int_{-\infty}^{\infty}|x|^{p r-1} f^{p}(x) \mathrm{d} x<\infty$, and $0<\int_{-\infty}^{\infty}|x|^{q r-1} g^{q}(x) \mathrm{d} x<\infty$, then

$$
\begin{aligned}
I^{*} & :=\int_{-\infty}^{\infty} \int_{-\infty}^{\infty} f(x) g(y)\left|\ln \frac{1+2 x y \cos \alpha+x^{2} y^{2}}{1+2 x y \cos \beta+x^{2} y^{2}}\right| \mathrm{d} x \mathrm{~d} y \\
& <k\left(\int_{-\infty}^{\infty}|x|^{p r-1} f^{p}(x) \mathrm{d} x\right)^{1 / p}\left(\int_{-\infty}^{\infty}|x|^{q r-1} g^{q}(x) \mathrm{d} x\right)^{1 / p}
\end{aligned}
$$

and

$$
\begin{aligned}
J & =\int_{-\infty}^{\infty}|y|^{-p r+p-1}\left(\int_{-\infty}^{\infty} f(x)\left|\ln \frac{1+2 x y \cos \alpha+x^{2} y^{2}}{1+2 x y \cos \beta+x^{2} y^{2}}\right| \mathrm{d} x\right)^{p} \mathrm{~d} y \\
& <k^{p} \int_{-\infty}^{\infty}|x|^{p r-1} f^{p}(x) \mathrm{d} x
\end{aligned}
$$

Inequalities (3.1)and (3.2) are equivalent, and the constant factors in the two forms are all the best possible.

Proof If (2.7) takes the form of equality for some $y \in(-\infty, 0) \cup(0, \infty)$, then there exist constants $M$ and $N$, such that they are not all zero, and

$$
M \frac{|x|^{r(p-1)}}{|y|^{r}} f^{p}(x)=\frac{|y|^{r(q-1)}}{|x|^{r}} \text { a.e. in }(-\infty, \infty) \times(-\infty, \infty),
$$

Hence, there exists a constant $C$, such that $M|x|^{r p} f^{p}(x)=N|y|^{r q}=C$ a.e. in $(-\infty, \infty) \times(-\infty, \infty)$.

We claim that $M=0$. In fact, if $M \neq 0$, then $|x|^{r p-1} f^{p}(x)=C /(M|x|)$ a.e. in $(-\infty, \infty)$ which contradicts the fact that $0<\int_{-\infty}^{\infty}|x|^{p r-1} f^{p}(x) \mathrm{d} x<\infty$. In the same way, we claim that $N=0$. This is too a contradiction and hence by (2.7), we have (3.2).

By Holder's inequality with weight and (3.2), we have, 


$$
\begin{aligned}
I^{*} & =\int_{-\infty}^{\infty}\left[|y|^{-r+1 / q} \int_{-\infty}^{\infty} f(x)\left|\ln \frac{1+2 x y \cos \alpha+x^{2} y^{2}}{1+2 x y \cos \beta+x^{2} y^{2}}\right| \mathrm{d} x\right]\left[|y|^{r-1 / q} g(y)\right] \mathrm{d} y \\
& \leq(J)^{1 / p}\left(\int_{-\infty}^{\infty}|y|^{q r-1} g^{q}(y) \mathrm{d} y\right)^{1 / q}
\end{aligned}
$$

Using (3.2), we have (3.1).

\section{Setting}

$$
g(y)=|y|^{-p r+p-1}\left(\int_{-\infty}^{\infty} f(x)\left|\ln \frac{1+2 x y \cos \alpha+x^{2} y^{2}}{1+2 x y \cos \beta+x^{2} y^{2}}\right| \mathrm{d} x\right)^{p-1}
$$

then $J=\int_{-\infty}^{\infty}|y|^{q r-1} g^{q}(y)$ dy by (2.7) we have $J<\infty$. if $J=0$ then (3.2) is proved; if $0<J<\infty$, by (3.1), we obtain

$$
\begin{gathered}
0<\int_{-\infty}^{\infty}|y|^{q r-1} g^{q}(y) \mathrm{d} y=J=I^{*} \\
<k\left(\int_{-\infty}^{\infty}|x|^{p r-1} f^{p}(x) \mathrm{d} x\right)^{1 / p}\left(\int_{-\infty}^{\infty}|x|^{q r-1} g^{q}(x) \mathrm{d} x\right)^{1 / q}, \\
\left(\int_{-\infty}^{\infty}|x|^{q r-1} g^{q}(x) \mathrm{d} x\right)^{1 / p}=J^{1 / p}<k\left(\int_{-\infty}^{\infty}|x|^{p r-1} f^{p}(x) \mathrm{d} x\right)^{1 / p}
\end{gathered}
$$

Inequalities (3.1) and (3.2) are equivalent.

If the constant factor $k$ in (3.1) is not the best possible, then there exists a positive $h$ (with $h<k$ ), such that

$$
\begin{aligned}
& \int_{-\infty}^{\infty} \int_{-\infty}^{\infty} f(x) g(y)\left|\ln \frac{1+2 x y \cos \alpha+x^{2} y^{2}}{1+2 x y \cos \beta+x^{2} y^{2}}\right| \mathrm{d} x \mathrm{~d} y \\
& <h\left(\int_{-\infty}^{\infty}|x|^{p r-1} f^{p}(x) \mathrm{d} x\right)^{1 / p}\left(\int_{-\infty}^{\infty}|x|^{q r-1} g^{q}(x) \mathrm{d} x\right)^{1 / q}
\end{aligned}
$$

For $\varepsilon>0$, by (3.4), using lemma 2.3, we have

$$
\begin{aligned}
\tilde{I}(\varepsilon) & =k+o(1) \\
& <\varepsilon h\left(\int_{-\infty}^{\infty}|x|^{p r-1} \mid f^{p}(x) \mathrm{d} x\right)^{1 / p}\left(\int_{-\infty}^{\infty}|x|^{q r-1} g^{q}(x) \mathrm{d} x\right)^{1 / q}
\end{aligned}
$$

Hence we find, $k+o(1)<h$, for $\varepsilon \rightarrow 0^{+}$, it follows that $k \leq h$, which contradicts the fact that $h<k$. Hence the constant $h$ in (3.1) is the best possible. As (3.1) and (3.2) are equivalent, if the constant factor in (3.2) is not the best possible, then by using (3.2), we can get a contradiction that the constant factor in (3.1) is not the best possible.

Thus we complete the prove of the theorem.

Remark For $\alpha=\frac{\pi}{4}, \beta=\frac{\pi}{3}$ in (3.1), we have the following particular result:

$$
\begin{aligned}
& \int_{-\infty}^{\infty} \int_{-\infty}^{\infty} f(x) g(y)\left|\ln \frac{1+\sqrt{2} x y+x^{2} y^{2}}{1+x y+x^{2} y^{2}}\right| \mathrm{d} x \mathrm{~d} y \\
& <k\left(\int_{-\infty}^{\infty}|x|^{p r-1} f^{p}(x) \mathrm{d} x\right)^{1 / p}\left(\int_{-\infty}^{\infty}|x|^{q r-1} g^{q}(x) \mathrm{d} x\right)^{1 / q}
\end{aligned}
$$

Where the constant factor

$$
k=\frac{4 \pi \sin \frac{(r-1) \pi}{24} \cos \frac{5(r-1) \pi}{24}}{(r-1) \sin \frac{\pi r}{2}} \text { also is the best possible. }
$$

\section{References}

[1] G. H. Hardy, J. E. Littlewood and G. Polya, "Inequalities," Cambridge University Press, Cambridge, 1952.

[2] G. H. Hardy, "Note on a Theorem of Hilbert Concerning Series of Positive Terems," Proceedings of London Mathematical Society, Vol. 23, No. 2, 1925, pp. 45-46.

[3] B. C. Yang, "A New Hilbert-Type Integral Inequality with a Parameters," Journal of Henan University (Natural Science), Vol. 35, No. 4, 2005, pp. 4-8.

[4] Z. T. Xie and Z. Zeng, "A Hilbert-Type Integral Inequality whose Kernel is a Homogeneous Form of Degree-3," Journal of Mathematical Analysis Application, Vol 339, No. 1, 2008, pp. 324-331.doi:10.1016/j.jmaa.2007.06.059

[5] B. C. Yang, "A New Hilbert-Type Integral Inequality with Some Parameters," Journal of Jilin University (Science Edition), Vol. 46, No. 6, 2008, pp. 1085-1090.

[6] B. C. Yang, "A Hilbert-Type Intergral Inequality with the Homogeneous Kernel of Real Number-Degree," Journal of Jilin University (Science Edition), Vol. 47, No. 5, 2009, pp. 887-892.

[7] Z. T. Xie and X. D. Liu, "A New Hilbert-Type Integral Inequality and its Reverse," Journal of Henan University, (Science Edition), Vol. 39, No. 1, 2009, pp. 10-13.

[8] Z. Zeng and Z. T. Xie, "On a New Hilbert-Type Integral Inequality with the Integral in Whole Plane," Journal of Inequalities and Applications, Vol. 2010, Article ID 256796, 2010.

[9] Z. T. Xie, B. C. Yang and Z. Zeng, “A New Hilbert- type Integral Inequality with the Homogeneous Kernel of Real Number-Degree," Journal of Jilin University (Science Edition), Vol. 48, No. 6, 2010, pp. 941-945.

[10] Z. T. Xie and Z. Zeng, "On Generality of Hilbert's Inequality with Best Constant Factor," Natural Science Journal of Xiangtan University, Vol. 32, No. 3, 2010, pp. $1-4$.

[11] Z. T. Xie and B. L. Fu, "A New Hilbert-Type Integral Inequality with a Best Constant Factor," Journal of Wuhan University (Natural Science Edition), Vol. 55, No. 6, 2009, pp. 637-640.

[12] Z. T. Xie and Z. Zeng, "The Hilbert-Type Integral Inequality with the System Kernel of $-\lambda$ Degree Homogeneous Form," Kyungpook Mathematical Journal, Vol. 
50, No. 2, 2010, pp. 297-306.

[13] Z. T. Xie and F. M. Zhou, "A Generalization of a Hilbert-Type Inequality with the Best Constant Factor," Journal of Sichuan Normal University (Natural Science), Vol. 32, No. 5, 2009, pp. 626-629.

[14] Z. T. Xie and Z. Zeng, "A Hilbert-Type Integral Inequality with a Non-Homogeneous Form and a Best Constant
Factor," Advances and Applications in Mathematical Sciens, Vol. 3, No. 1, 2010, pp. 61-71.

[15] Z. Zeng and Z. T. Xie, "A New Hilbert-Type Integral Inequality with a Best Constant Factor," Journal of South China Normal University (Natural Science Edition), Vol. 3, 2010, pp. 31-33. 